\title{
Die Wirkung von Monojodessigsäure auf Atmung und Photosynthese von Chlorella*
}

\author{
Von O. Kandler, I. Liesenkötter und B. A. OAKs ** \\ Aus dem Bakteriologischen Institut der Südd. Versuchs- und Forschungsanstalt für Milchwirtschaft, \\ Weihenstephan \\ (Z. Naturforschg. 16 b, 50 - 61 [1961] ; eingegangen am 20. Oktober 1960)
}

\begin{abstract}
The distribution of radioactivity after feeding labeled glucose, as well as the change of the poolsize of intermediates, in Chlorella poisoned with monoiodacetic acid (MIA), showed that tric.sephosphate dehydrogenase is the most sensitive Enzyme in respiration. MIA leads to a large accumulation of fructose diphosphate (FDP) and a large decrease in phosphoglyceric acid (PGA). Also, the breakdown of glucose via 6-phospho-gluconate is inhibited by MIA, as shown by the small change of the $\mathrm{C}_{1} / \mathrm{C}_{6}$ ratio.

FDP piles up during MIA poisoning in the dark and is quickly transformed to ribulose diphosphate (RuDP), in the light; subsequently, RuDP is transformed to PGA and its derivatives (pyruvate and other acids) by carboxylation and hydrolysis of the ketoacid. Thus, a third pathway of glucose breakdown, by passing glycolysis and the 6-phosphogluconate pathway, is induced by MIA in the light.

The photosynthetic $\mathrm{CO}_{2}$ assimilation of Chlorella is about 100 fold more sensitive toward MIA than respiration. By feeding labeled $\mathrm{CO}_{2}$ and determining the concentration of the intermediates formed during MIA poisoning in the light, it is found that, in contrast to the behavior of broken chloroplasts, the reduction of the fixed $\mathrm{CO}_{2}$ is the most sensitive reaction of photosynthesis in Chlorella, rather than the formation of RuDP.

It is assumed that the energy-rich phosphate formed in the light may be used for a relatively MIAinsensitive reaction leading to the formation of RuDP, and that the carboxylated RuDP is directly reduced to sugar by a highly MIA-sensitive reaction. Hydrolysis to PGA occurs only if the reduction of the ketoacid proceeds too slowly, i. g., during MIA-inhibition.
\end{abstract}

Nach dem von Calvin und Mitarb. ${ }^{1}$ vorgeschlagenen und heute am meisten akzeptierten Schema der Photosynthese wird die Reduktion des Kohlenstoffes zur Kohlenhydratstufe in Umkehrung der Glykolyse nach Gl. (1) formuliert.

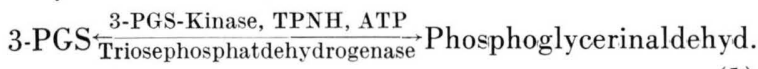

Da Monojodessigsäure (MJ) ein starker Hemmstoff für die Triosephosphatdehydrogenase ist, sollte bei Vergiftung der Photosynthese mit diesem Gift die Reduktion unterbunden und Phosphoglycerinsäure (PGS) angereichert werden. Die schon sehr frühzeitig ausgeführten Versuche von StepкA ${ }^{2}$ (vgl. auch Calvin und Massini ${ }^{3}$ ) mit ${ }^{14} \mathrm{CO}_{2}$ ergaben aber den gegenteiligen Effekt. Die Hemmung betraf die PGS stärker als die Zucker und er nahm daher an, $\mathrm{da} 3$ die photosynthetische $\mathrm{CO}_{2}$-Reduktion nicht mit der Umkehr der Glykolyse identisch ist.

* Herrn Professor Kurt Mothes zum 60. Geburtstag gewidmet.

** Humboldt-Stipendiat 1959/60. Gegenwärtige Anschrift: Department of Biological Sciences, Purdue University, Lafayette/Indiana.

1 J. A. Bassham u. M. Calvin, The path of carbon in Photosynthesis. Englewood Cliffs, N. J. 1957.
Auch Newburgh und Burris ${ }^{4}$, die bei MJ-vergifteten Blättern ${ }^{32} \mathrm{P}$ verabfolgten, stellten fest, daß die PGS nicht angestaut wurde. Sie fanden sogar, daß die spezifische Aktivität der Zuckerphosphate bei den vergifteten Proben erheblich höher war als die der PGS und schlossen daraus, daß die Photosynthese unter der MJ-Wirkung unter Umgehung der Glykolyse verläuft.

An gereinigten Enzym-Präparationen von Triosephosphatdehydrogenase und Ribulose-5-Phosphatkinase konnten Calo und Gibbs ${ }^{5}$ neuerdings zeigen, daß die Kinase gegen MJ um eine Zehnerpotenz empfindlicher ist als die DPN und TPN abhängigen Dehydrogenasen. Sie untersuchten außerdem die Empfindlichkeit der $\mathrm{CO}_{2}$-Fixierung ganzer Chloroplasten und stellten fest, daß diese etwa gleich war mit der Empfindlichkeit der Ribulose-5-Phosphatkinase.

2 W. Stepka, Doktoral Dissertation, Univ. of California, Berkeley 1951.

3 M. Calvin u. P. Massini, Experientia [Basel] VIII, 445 [1952].

${ }^{4}$ R. W. Newburgh u. R. H. Burris, Arch. Biochem. Biophysics 49, 98 [1954].

5 N. Calo u. M. Gibbs, Z. Naturforschg. 15 b, 287 [1960]. 
Trebst und Mitarbb. ${ }^{6}$ analysierten die Wirkung von $\mathrm{MJ}$ auf die $\mathrm{CO}_{2}$-Fixierung isolierter ,gebrochener" Chloroplasten. Verwendeten sie Ribose-5-Phosphat als $\mathrm{CO}_{2}$-Acceptor, so ergaben schon $10^{-3}-\mathrm{m}$. MJ 70\% Hemmung, während bei Verwendung von Ribulosediphosphat erst $10^{-2}-m$. MJ zu $36 \%$ hemmten. Im letzteren Fall war dann der Anteil der PGS an der Aktivität der Fixierungsprodukte deutlich erhöht. Die Verfasser schließen daraus, daß bei der Photosynthese nicht die Triosephosphatdehydrogenase, sondern die Ribulose-5-phosphatkinase das gegen MJ empfindlichste Ferment ist. Damit sind die Befunde von Stepka ${ }^{2}$ bzw. Newburgh und Burris ${ }^{4}$ (mit Ausnahme der Inversion der spezifischen Aktivitäten) erklärbar.

$\mathrm{Zu}$ gegensätzlichen Befunden gelangten jedoch Simonis und Weichart ${ }^{7}$, die bei MJ-Vergiftung von Elodea-Blättchen unter Verwendung von ${ }^{32} \mathrm{P}$ eine starke Ansammlung von PGS und Phosphoenolbrenztraubensäure (PEB), aber eine Verminderung der Zuckerphosphate fanden. Sie schlossen daraus, daß die Triosephosphat-Reduktion der Angriffspunkt der Photosynthese-Hemmung in vivo ist. Eigene Versuche ${ }^{8}$ über die Verteilung der Radioaktivität nach Fütterung von ${ }^{14} \mathrm{CO}_{2}$ zu MJ vergifteten Chlorellen hatten ebenfalls eine Anreicherung von PGS ergeben. Im folgenden sollen ausführliche vergleichende Versuche über die Wirkung von MJ auf die Verteilung der Aktivität nach Fütterung von markiertem $\mathrm{CO}_{2}$ bzw. Glucose und auf die Veränderung der Zwischenstoff-Konzentrationen bei Atmung und Photosynthese mitgeteilt werden.

\section{Material und Methodik}

Es wurden 2 Arten von Chlorella benützt; der schon früher verwendete Stamm von Chlorella pyrenoidosa $\left(\mathrm{K}_{\text {ANDLEER }}{ }^{9}\right.$ ) und ein Stamm von Chlorella elipsoidea, der uns von Prof. PIrson überlassen wurde. Die Anzucht erfolgte in $1-l$-Waschflaschen unter Durchlüftung mit rund $5 \% \mathrm{CO}_{2}$ in Luft und Beleuchtung mit Leuchtstoffröhren. Die Nährlösung war die früher l. c. ${ }^{9}$ beschriebene. Geerntet wurde in 3- bis 5-tägigen Abständen. Stammkulturen wurden auf Schrägagar gehalten.

Die manometrischen Messungen wurden in üblicher Weise durchgeführt, wobei zur Beleuchtung eine XenonHochdrucklampe entsprechend der früheren Beschreibung ${ }^{10}$ Verwendung fand.

6 A. V. Trebst, M. Cosada u. D. J. Arnon, J. biol. Chemistry 235, 840 [1960].

7 W. Simonis u. G. Weichart, Z. Naturforschg. 13 b, 694 [1958].
Kurzfristige Photosynthese-Versuche wurden in einem „Lollipop“ durchgeführt, der in einem Kühlmantel eingeschmolzen war. Die Kühlung erfolgte mit Wasser, das einem Thermostaten entnommen wurde. Die ${ }^{14} \mathrm{CO}_{2}$ Fixierung und die chromatographische Aufarbeitung des Materials wurde in der früher beschriebenen Weise ${ }^{11}$ durchgeführt.

Bei Fütterung von markierter Glucose wurde das gleiche Verfahren verwendet und lediglich ${ }^{14} \mathrm{CO}_{2}$ durch Glucose ersetzt. Langfristige Versuche wurden in den Manometriegefäßen durchgeführt und die Algen durch Zuführung von Alkohol durch den Belüftungskamin des seitlichen Ansatzes abgetötet und wie üblich aufgearbeitet.

Zur Bestimmung der Zwischenstoff-Konzentrationen wurden die Algen nicht in Alkohol, sondern in Trichloressigsäure (6\% Endkonzentration) abgetötet und zweimal mit 5-proz. Trichloressigsäure ausgewaschen. Die im folgenden angeführten enzymatischen Bestimmungen wurden direkt in dem durch Zentrifugieren geklärten Extrakt ausgeführt. Lediglich beim Nachweis von Glucose-6-phosphat war es notwendig, die Trichloressigsäure durch Ätherextraktion zu entfernen. Die notwendigen Enzyme wurden von der Firma Boehringer, Mannheim, bezogen.

Die Reaktionsmischungen für die optischen Teste hatten folgende Zusammensetzung:

1. Adenosintriphosphat (ATP). Angabe in ml: 0,15 DPNH $(372 \gamma), 0,3$ 0,1-m. Hydrazinsulfat, 0,1 1,2-m. Cystein HCl, 0,19 1-m. KOH, 0,6 1-m. Glykokoll, 0,12 0,5-m. $\mathrm{MgSO}_{4}, 0,6$ 0,1-m. PGS, 1,5 Extrakt, 2,14 0,2-m. Trapp Puffer, $p_{\mathrm{H}}$ 7,6, (Triäthanolamin), $\quad 0,2$ Glycerinaldehydphosphatdehydrogenase $(100 \gamma), 0,1 \quad 3$-Phosphoglyceratkinase $(60 \gamma)=6 \mathrm{ml}$; $p_{\mathrm{H}} 7,6 ; d=4$.

2. PGS wie für ATP, aber PGS wird durch $0,6 \mathrm{ml}$ ATP ersetzt.

3. Phosphoglycerinaldehyd (GPA) und Dihydroxyaceton phosphat (DAP). A n g abe in ml: 0,1 DPNH, 0,12 Trilon, 0,3 0,27-m. Glykokoll, 0,6 0,1-m. Cystein HCl, 0,1 1-m. KOH, 1,5 Extrakt, 2,92 Trapp Puffer, 0,2 Glycerophosphatdehydrogenase/Trioseisomerase $(=100 \gamma)$.

4. Fruktosediphosphat (FDP), wie für DAP und GAP, aber Zugabe von $0,1 \mathrm{ml}$ Aldolase $(50 \gamma)$.

5. Brenztraubensäure (BTS). Angabe i n ml: $0,15 \mathrm{DPNH}, 0,122-m$. KCl, 0,1 0,5-m. $\mathrm{MgSO}_{4}$, 0,16 Trilon, 1,5 Extrakt, 3,84 Trapp Puffer, 0,1 Milchsäuredehydrogenase $(50 \gamma)$.

6. Phosphoenolbrenztraubensäure (PEB), wie für BTS, aber Zugabe von $0,2 \mathrm{ml}$ 0,02-m. ADP und $0,1 \mathrm{ml}$ Pyruvatkinase $(50 \gamma)$.

7. Adenosindiphosphat (ADP), wie für PEB, aber statt ADP, Zugabe von 0,2 ml 0,025-m. PEB.

8 O. Kandler, Proc. IX. Int. Bot. Congress Vol. II, S. 184 (1959).

9 O. Kandler, Z. Naturforschg. 5 b, 423 [1950].

10 O. Kandler u. F. Schötz, Z. Naturforschg. 11 b, 708 [1956].

11 O. Kandler, Z. Naturforschg. 13 b, 219 [1958]. 


\begin{tabular}{|c|c|c|c|c|c|c|c|}
\hline \multicolumn{2}{|c|}{ MJ-Konzentration } & & $10^{-6}-m$ & $10^{-5}-m$ & $3 \cdot 10^{-5}-m$ & $10^{-4}-m$. & $3 \cdot 10^{-4}-m$. \\
\hline \multirow{3}{*}{ 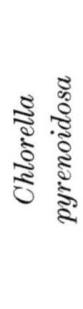 } & Leeratmung & $\begin{array}{l}\mathrm{a} \\
\mathrm{b} \\
\mathrm{c}\end{array}$ & $\begin{array}{l}\overline{96} \\
-\end{array}$ & $\begin{array}{r}100 \\
104 \\
-\end{array}$ & $\begin{array}{r}105 \\
99 \\
74\end{array}$ & $\begin{array}{l}78 \\
87 \\
54\end{array}$ & $\begin{array}{l}68 \\
80 \\
48\end{array}$ \\
\hline & Glucoseatmung & $\begin{array}{l}\mathrm{a} \\
\mathrm{b} \\
\mathrm{c}\end{array}$ & $\begin{array}{r}\overline{101} \\
-\end{array}$ & $\begin{array}{r}103 \\
101 \\
-\end{array}$ & $\begin{array}{r}110 \\
103 \\
53\end{array}$ & $\begin{array}{l}59 \\
75 \\
27\end{array}$ & $\begin{array}{l}49 \\
70 \\
20\end{array}$ \\
\hline & $\begin{array}{l}\mathrm{CO}_{2} \text {-Fixierung } \\
\text { (Photosynthese) }\end{array}$ & $\begin{array}{l}\mathrm{a} \\
\mathrm{b} \\
\mathrm{c}\end{array}$ & $\begin{array}{l}22 \\
29 \\
-\end{array}$ & $\begin{array}{r}2 \\
2 \\
23\end{array}$ & $\begin{array}{c}- \\
1 \\
2\end{array}$ & $\frac{-}{1}$ & $\begin{array}{l}- \\
- \\
-\end{array}$ \\
\hline \multirow{3}{*}{ 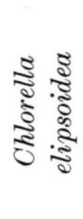 } & Leeratmung & $\begin{array}{l}\mathrm{a} \\
\mathrm{b}\end{array}$ & $\begin{array}{c}106 \\
-\end{array}$ & 111 & $\begin{array}{l}118 \\
110\end{array}$ & $\begin{array}{r}105 \\
72\end{array}$ & $\begin{array}{l}- \\
-\end{array}$ \\
\hline & Glucoseatmung & $\begin{array}{l}\mathrm{a} \\
\mathrm{b}\end{array}$ & 101 & $\begin{array}{l}96 \\
-\end{array}$ & $\begin{array}{l}93 \\
90\end{array}$ & $\begin{array}{l}91 \\
86\end{array}$ & $\begin{array}{l}84 \\
-\end{array}$ \\
\hline & $\begin{array}{l}\mathrm{CO}_{2} \text {-Fixierung } \\
\text { (Photosynthese) }\end{array}$ & $\begin{array}{l}\mathrm{a} \\
\mathrm{b}\end{array}$ & $\frac{95}{-}$ & $\begin{array}{l}25 \\
77\end{array}$ & $\begin{array}{r}8 \\
34\end{array}$ & $\begin{array}{l}1 \\
2\end{array}$ & 1 \\
\hline
\end{tabular}

Tab. 1. Vergleich von $\mathrm{O}_{2}$-Verbrauch und ${ }^{14} \mathrm{CO}_{2}$-Assimilation nach 2 Stdn. Vorvergiftung mit MJ. Kontrolle=100 Prozent. Bei $\mathrm{a}, \mathrm{b}$ und $\mathrm{c}$ handelt es sich um Parallelproben mit Algen verschiedener Ernte. $m / 30$-Phosphatpuffer $p_{\mathrm{H}} 5,5$. Für die Photosynthesemessung Zusatz von $4 \mu$ Molen $\mathrm{KH}^{14} \mathrm{CO}_{2}$. Atmungsmessung $2 \mathrm{Stdn}$. Photosynthese 1 Stunde.

8. Glucose-6-phosphat. Angabe in ml: $2 \mathrm{H}_{2} \mathrm{O}, 2$ Tris-Puffer, $p_{\mathrm{H}} 8$, (Trioxymethylaminomethan), 0,2 0,3-m. $\mathrm{MgCO}_{2}, 0,2 \mathrm{TPN}, 1,5$ Extrakt, 0,1 Zwischenferment $(10 \gamma)$.

Die jeweils in einer Tabelle bzw. Abbildung zusammengefaßten Daten wurden stets durch Analyse aliquoter Teile desselben Extraktes erhalten. Zur Gewinnung derart großer Extraktmengen wurden für jede Probe $5 \mathrm{ml}$ einer 28-proz. Suspension entnommen.

\section{Versuchsergebnisse}

I. Vergleich der Empfindlichkeit von Atmung und Photosynthese

Schon KohN ${ }^{12}$ und Holzer ${ }^{13}$ konnten zeigen, daß die endogene Atmung von Chlorella von MJ weniger gehemmt wird als die Photosynthese. Die in Tab. 1 zusammengefaßten Ergebnisse der eigenen Versuche zeigen diesen Sachverhalt ebenfalls. Darüber hinaus geht daraus hervor, daß die Glucoseatmung deutlich empfindlicher als die endogene Atmung, aber erheblich resistenter als die Photosynthese ist. Der Vergleich der beiden Algenarten zeigt, daß Chl. elipsoidea unempfindlicher ist als Chl. pyrenoidosa. Innerhalb der verschiedenen Ernten ergeben sich Unterschiede in der Empfindlichkeit, die einer halben Zehnerpotenz entsprechen.

Konzentrationen, die die Atmung noch nicht hemmen, führen häufig zu leichten Atmungssteigerungen,

12 H. J. Koнn, J. gen. Physiol. 19, 23 [1935]. die jedoch nicht regelmäßig reproduziert werden konnten.

Zur Methodik der in Tab. 1 zusammengefaßten Versuche sei folgendes bemerkt: Die Algen wurden vor dem Versuch zweimal in dest. Wasser gewaschen und nach Suspendieren in Phosphatpuffer, $p_{\mathrm{H}} 5,5$, $2-3$ Stdn. mit den entsprechenden Konzentrationen MJ unter Schütteln im Dunkeln inkubiert, um eine konstante Giftwirkung zu erhalten. AnschlieBend wurden die Algen auf die W a r b u r g - Gefäße verteilt und die manometrische Atmungsmessung begonnen. Zur Bestimmung der Photosynthese wurde in den Seitenarm $4 \mu$ Mole $\mathrm{KH}^{14} \mathrm{CO}_{3}$ gefüllt und die Algen mit $\mathrm{HCl}$ so stark angesäuert, daß nach Einkippen des Bicarbonats wieder $p_{\mathrm{H}} 5,5$ hergestellt war. Die Photosynthese wurde durch Messung der Radioaktivität der Algen nach einer Stde. Belichtung im Bereich der Lichtsättigung bestimmt. Alle 3 Stoffwechselleistungen wurden jeweils an der gleichen Algensuspension zur gleichen Zeit bestimmt, so daß die mit gleichen Buchstaben ( $a, b, c)$ bezeichneten Versuche in sich vergleichbar sind.

II. Die qualitative Wirkung auf die A t mung

\section{a) Das $C_{1} / C_{6}$-Verhältnis unter $M J$}

Wenn bei der Atmung durch MJ die Triosephosphatdehydrogenase gehemmt wird, so ist zu erwar-

${ }^{13}$ H. Holzner, Angew. Chem. 66, 65 [1954]. 
ten, daß der davon unabhängige oxydative Glucoseabbau über 6-Phosphogluconsäure relativ gefördert wird. Die anfallende Triose könnte wieder zur Hexose kondensiert und im Extremfall ein kompletter Glucoseabbau ohne Glykolyse erzwungen werden.

Der einfachste Weg, eine Verschiebung in der Aktivität der beiden Abbauwege festzustellen, ist die Bestimmung des Verhältnisses zwischen dem aus $\mathrm{C}_{1}$ und $_{6}$ der Glucose entstehenden $\mathrm{CO}_{2}$. Daher wurden zu Parallelproben von 24 Stdn. verarmten Algen (Chl. pyrenoidosa) Glucose-[1-14 C] bzw. Glucose- $\left[6-{ }^{14} \mathrm{C}\right]$ gegeben, in einem 2-Stdn.-Intervall das entstehende $\mathrm{CO}_{2}$ aufgefangen und dessen Akti-

\begin{tabular}{|c|c|c|c|c|c|}
\hline MJ-Konzentration & $K$ & $\begin{array}{c}\stackrel{\dot{\xi}}{1} \\
\text { bे } \\
0\end{array}$ & $\begin{array}{c}\dot{\hat{g}} \\
\dot{1} \\
\dot{0} \\
\dot{\infty} \\
\dot{\infty}\end{array}$ & $\begin{array}{l}\stackrel{\xi}{1} \\
\stackrel{1}{1}\end{array}$ & $\begin{array}{l}\dot{E} \\
\dot{1} \\
\stackrel{1}{0} \\
\dot{\infty}\end{array}$ \\
\hline $\begin{array}{l}\mathrm{C}-1 / \mathrm{C}-6 \\
\mathrm{O}_{2}-\text { Verbrauch }[\%]\end{array}$ & $\begin{array}{l}2,06 \\
100\end{array}$ & $\begin{array}{l}2,25 \\
101\end{array}$ & $\begin{array}{c}2,29 \\
91\end{array}$ & $\begin{array}{c}2,13 \\
77\end{array}$ & $\begin{array}{c}2,05 \\
65\end{array}$ \\
\hline
\end{tabular}

Tab. 2. $\mathrm{O}_{2}$-Verbrauch und $\mathrm{C}_{1} / \mathrm{C}_{6}$-Verhältnis von Chlorella pyrenoidosa nach 2 Stdn. Vorvergiftung mit MJ. m/30-Phosphatpuffer $p_{H} 5,5$. Versuchsdauer 2 Stunden.

vität bestimmt. Wie die Tab. 2 zeigt, entsteht bei den unvergifteten Proben doppelt so viel $\mathrm{CO}_{2}$ aus der 1-Position als aus der 6-Position. Die direkte Oxy- dation zur 6-Phosphogluconsäure spielt also bei Chlorella neben der normalen Glykolyse eine erhebliche Rolle. Zusatz von MJ führt bei Konzentrationen, die die Glucoseatmung nicht oder nur schwach hemmen, zu einer leichten Erhöhung des $C_{1} / C_{6}$. Quotienten. Bei weiterer Konzentrations-Erhöhung und verstärkter Atmungshemmung verschwindet die Erhöhung wieder. Man kann annehmen, daß das Einsetzen der Hemmung der Triosephosphatdehydrase bei $10^{-5}-m$. MJ zunächst durch Verstärkung der direkten Oxydation zu 6-Phosphogluconat kompensiert und damit die Verminderung des $\mathrm{O}_{2}$-Verbrauchs ausgeglichen, bei der endogenen Atmung häufig sogar überkompensiert wird. Offenbar ist aber diese über TPN/TPNH verlaufende Oxydation mit geringerer Phosphorylierung verbunden, wie der Abfall der oxydativen Glucoseassimilation (Kandler ${ }^{14}$ ) zeigt. Mit zunehmender Konzentrations-Erhöhung wird der direkte Abbauweg ebenfalls gehemmt und der $\mathrm{C}_{1} / \mathrm{C}_{6}$-Quotient normalisiert sich.

\section{b) Lokalisierung der MJ-Hemmung}

Die Lokalisierung der Unterbrechung einer Reaktionskette durch einen Hemmstoff läßt sich am besten durch die Bestimmung der Veränderungen in den Zwischenstoff-Konzentrationen durchführen. Da-

\begin{tabular}{|c|c|c|c|c|c|}
\hline \multirow[b]{2}{*}{ MJ-Konzentration } & \multicolumn{2}{|c|}{ Dunkel } & \multicolumn{2}{|c|}{ Licht } & \\
\hline & Kontrolle & $\begin{array}{l}10^{-4}-m . \\
\text { MJ }\end{array}$ & Kontrolle & $\begin{array}{l}10^{-4}-\mathrm{m} . \\
\mathrm{MJ}\end{array}$ & \\
\hline $\begin{array}{l}\text { Gesamtassimilation in \% der } \\
\text { Dunkel-Kontrolle }\end{array}$ & 100 & 52 & 130 & 135 & \\
\hline $\begin{array}{l}\text { Unlöslich } \\
\text { (Polysaccharid) }\end{array}$ & 21 & 21,5 & 68,0 & 68 & $\begin{array}{l}\text { Radioaktivität in \% der } \\
\text { assimilierten Glucose }\end{array}$ \\
\hline Saccharose & 39 & 34,7 & 14,0 & 12,5 & \\
\hline Phosphat-Ester & 15 & 16,1 & 7,0 & 9 & \\
\hline Org. Säuren und Aminosäuren & 25 & 21,7 & 11,0 & 10,4 & \\
\hline Diphosphate & 6,5 & 41,0 & 6,0 & 9,0 & Prozentuale Verteilung der \\
\hline Zuckermonophosphate & 65,0 & 45,0 & 50,0 & 40,0 & Radioaktivität innerhalb der \\
\hline UDPG * & 15,0 & 11,0 & 29,0 & 30,0 & Phosphatester \\
\hline PGS & 9,0 & 2,5 & 12,0 & 18,0 & \\
\hline PEB & 5,0 & 0,5 & 3,0 & 3,0 & \\
\hline Glucose und Heptulose & 70,0 & 56,0 & 63,0 & 59,0 & Verteilung der Radioaktivität \\
\hline Fructose & 17,0 & 38,0 & 17,0 & 13,0 & innerhalb der aus den \\
\hline Pentose & 1,0 & 1,0 & 4,0 & 4,0 & Phosphatestern gewonnenen \\
\hline Glycerinsäure & 9,0 & 2,5 & 12,0 & 18,0 & Verbindungen \\
\hline Rest & 3,0 & 2,5 & 4,0 & 6,0 & \\
\hline
\end{tabular}

Tab. 3. Verteilung der Radioaktivität nach 10 min Fütterung von radioaktiver Glucose zu Chlorella pyrenoidosa. Vorvergiftung 2 Stdn. $m / 100$-Phosphatpuffer $p_{\mathrm{H}} 5,5$. Beleuchtungsstärke 30000 Lux an beiden Seiten eines Lollipop. Gasphase Luft $0,1 \% \mathrm{CO}_{2}$. * Uridindiphosphoglucose.

14 O. KANDLER, Z. Naturforschg. 10 b, 37 [1955]. 


\begin{tabular}{|c|c|c|c|c|c|c|c|c|}
\hline \multirow{2}{*}{$\begin{array}{l}\text { MJ-Konzen- } \\
\text { tration }\end{array}$} & \multicolumn{4}{|c|}{ Leeratmung } & \multicolumn{4}{|c|}{ Glucoseatmung } \\
\hline & Kontrolle & $10^{-3}-m$ & $10^{-4}-m$ & $10^{-5}-m$ & Kontrolle & $10^{-3}-m$. & $10^{-4}-m$ & $10^{-5}-m$. \\
\hline ATP & 2,79 & 1,72 & 2,5 & 2,8 & 1,55 & 0,49 & 1,9 & 1,55 \\
\hline $\mathrm{ADP}$ & 1,75 & 1,98 & 1,7 & 1,71 & 1,63 & 0,72 & 1,47 & 1,52 \\
\hline PGS & 8,53 & 5,31 & 6,74 & 9,2 & 6,86 & 5,07 & 5,2 & 6,5 \\
\hline FDP & 0,199 & 4,85 & 3,0 & 0,23 & 0,34 & 7,5 & 4,92 & 1,3 \\
\hline DAP & & & & & & & & \\
\hline GAP & 0,46 & 1,55 & 0,95 & 0,4 & 0,84 & 2,04 & 2,3 & 0,98 \\
\hline BTS & 0,2 & 0,06 & 0,19 & 0,2 & 0,38 & 0,0 & 0,2 & 0,2 \\
\hline PEB & 1,46 & 00 & 0,51 & 1,45 & 0,32 & 0,069 & 0,07 & 0,32 \\
\hline $\begin{array}{c}\mathrm{O}_{2}-\text { Verbrauch } \\
{[\%]}\end{array}$ & 100 & 90 & 98 & 100 & 100 & 20 & 60 & 100 \\
\hline
\end{tabular}

Tab. 4. Zwischenstoff-Konzentrationen von Chlorella pyrenoidosa (bezogen auf die abzentrifugierte Algenmasse) nach 2-stdg. Einwirkung verschiedener MJ-Konzentrationen. Gasphase Luft. $m / 100$-Phosphatpuffer $p_{H} 5,5$. Werte $\cdot 10^{-4}-m$.

zu stehen 2 Methoden zur Verfügung, die beide angewendet wurden.

1. Uniform markierte Glucose wurde zu verarmten Algen gegeben, die unbehandelt oder $2 \mathrm{Stdn}$. mit $10^{-4}-m$. MJ vorvergiftet waren. Nach $10 \mathrm{~min}$ wurden die Algen durch Eingießen in heißen Alkohol abgetötet. Wie die Tab. 3 zeigt, ergeben sich die größten Unterschiede der Verteilung der Radioaktivität in den Phosphatestern. Während der Aktivitätsanteil von PGS und PEB um ein Vielfaches vermindert ist, steigt der der Diphosphate erheblich an. Die Analyse der Einzelkomponenten der Phosphatester zeigt, daß besonders die Fructose einen höheren Anteil aufweist, nicht aber die Pentose. Offensichtlich liegt die Hemmung der MJ zwischen PGS, die nicht mehr so rasch gebildet werden kann und dem FDP, das sich anreichert.

2. In Trichloressigsäure-Extrakten von Algen verschiedener Vorvergiftung wurden mit Hilfe der eingangs beschriebenen enzymatischen Teste die Zwischenstoff-Konzentrationen direkt gemessen. Tab. 4 zeigt das Ergebnis eines Versuchs, bei dem Proben einer verarmten Algensuspension ohne bzw. mit Glucosezusaiz mit verschiedenen Konzentrationen MJ für 2 Stdn. im Dunkeln inkubiert und dann extrahiert wurden. Am deutlichsten sind die Zunahme des FDP und des Triosephosphats sowie die Abnahme der PGS und deren Folgeprodukte PEB und BTS, sowohl bei der endogenen als auch bei der Glucoseatmung. Überraschend ist, daß die ZwischenstoffKonzentrationen schon drastische Änderungen zeigen, obwohl die Sauerstoffaufnahme bei der endogenen Atmung dieses stark verarmten Algenmaterials sehr wenig gehemmt wurde ( $10 \%$ Hemmung bei $10^{-3}-m$. MJ!). Vermutlich dienten bei diesen verarmten Algen als Atmungsmaterial nicht nur
Zucker, sondern vorwiegend andere Substrate. Auch bei der Glucoseatmung ergeben sich in Übereinstimmung mit der Erhöhung des $\mathrm{C}_{1} / \mathrm{C}_{6}$-Quotienten die ersten Anzeichen einer Hemmung schon bei der für den Sauerstoffverbrauch unwirksamen Konzentration von $10^{-5}-m$.

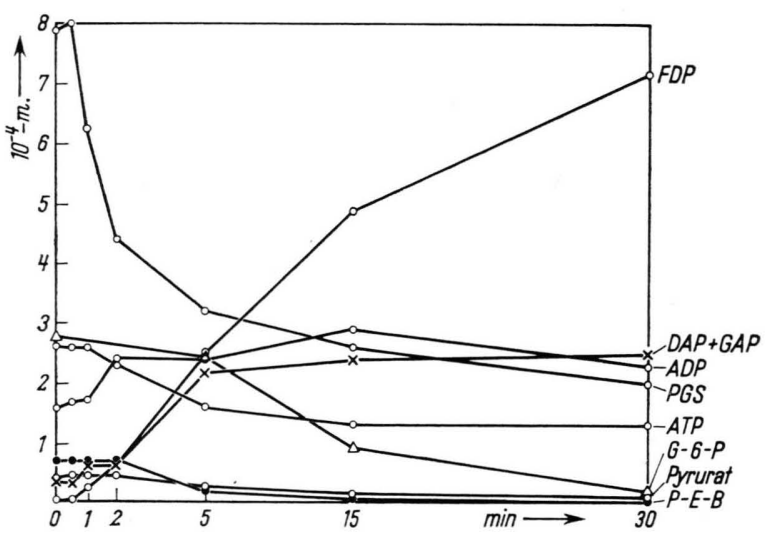

Abb. 1 a. Veränderung der Zwischenstoff-Konzentrationen bei Chlorella pyrenoidosa nach Zugabe von $3 \cdot 10^{-4}-m$. MJ nach 1-stdg. Inkubation mit $2 \%$ Glucose. Gasphase Luft. $m / 30$-Phosphatpuffer $p_{\mathrm{H}} 5,5$.

Die Kinetik der MJ-Wirkung zeigt Abb. $1 \mathrm{a}$ und $\mathrm{b}$. Bei diesem Versuch wurden verarmte Algen 1 Stde. mit $1 \%$ Glucose inkubiert um konstante Glucoseatmung zu erreichen und dann mit $3 \cdot 10^{-4}-m$. MJ versetzt. Der drastische Atmungsfall setzte nach 4 min ein (Abb. 1 b), während die Veränderungen der Zwischenstoff-Konzentrationen schon nach 1 min meßbar waren. Der normalerweise kleine Pool an FDP wurde in diesem Versuch zum weitaus größten Pool in der Zelle, während PGS und Folgeprodukte stark vermindert wurden. 


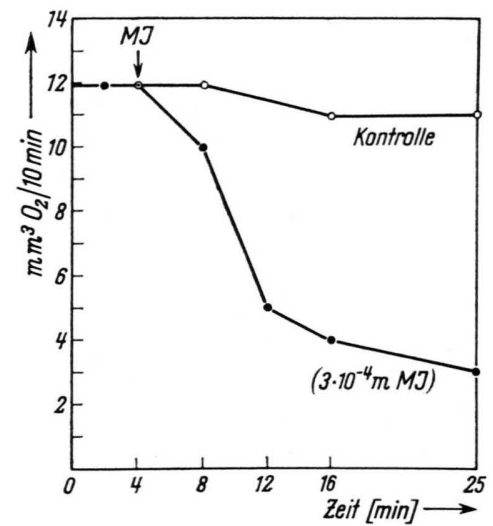

Abb. 1 b. Sauerstoffverbrauch von Chlorella pyrenoidosa nach Zugabe von $3 \cdot 10^{-4}-m$. MJ. Messung an Parallelproben der in Abb. 1 a benützten Suspension.

\section{Die qualitative Wirkung auf die Photosynthese}

Die Frage, ob MJ bei der Photosynthese die Lichtphosphorylierung oder den Weg des Kohlenstoffes betrifft, ist bereits seit längerer Zeit zugunsten der zweiten Möglichkeit entschieden, da sowohl bei ganzen Zellen (KANDLER ${ }^{14}$ ) als auch bei isolierten Chloroplasten (ARNoN ${ }^{15}$ ) gezeigt werden konnte, daß die Lichtphosphorylierung durch MJ nicht gehemmt wird.

Es besteht jedoch, wie einleitend betont, Unklarheit, ob in vivo die Hemmung der Triosephosphatdehydrogenase oder der Ribulose-5-phosphatkinase (Ru-5-P-Kinase) den begrenzenden Faktor für die $\mathrm{CO}_{2}$-Assimilation darstellt. a) Lokalisierung der MJ-Hemmung bei der Photosynthese durch Verwendung von ${ }^{14} \mathrm{CO}_{2}$.

Zunächst wurde die Verteilung der Radioaktivität nach 1 Stde. Photosynthese in ${ }^{14} \mathrm{CO}_{2}$ nach $2 \mathrm{Stdn}$. Vorvergiftung bestimmt. Der Versuchsansatz war der gleiche wie bereits bei der Messung der Gesamtfixierung (Tab. 1) beschrieben. Am Ende des Versuchs wurde der Alkohol in die Manometriegefäße gegeben, so daß sich 70\% Alkohol als Endkonzentration ergab.

Wie die Tab. 5 zeigt, wird vor allen Dingen die Zuckersynthese von MJ gehemmt, während die Aminosäuren einen erheblich höheren Anteil der Radioaktivität enthalten. Die bei der schwächeren Konzentration starke Erhöhung der Aktivität in den Phosphatestern ist ausschließlich auf die Zunahme der PGS zurückzuführen, während der Anteil der Zuckerphosphate erheblich kleiner wurde. Auch in der unlöslichen Fraktion in der bei den Kontrollen über $80 \%$ der Aktivität in Polysaccharid enthalten ist, nimmt unter MJ-Einfluß der Zuckeranteil ab und bei der stärksten Hemmung handelt es sich fast nur um radioaktive Aminosäuren, die zu Protein aufgebaut wurden. In der löslichen Fraktion tritt bei starker Hemmung Citrullin durch einen relativ hohen Aktivitätsanteil hervor, während es bei den Kontrollen stets nur Bruchteile von $\%$ ausmacht. Dies beruht darauf, daß die Carboxylierung von Ornithin zu Citrullin ${ }^{16}$ im Gegensatz zur photosynthetischen $\mathrm{CO}_{2}$-Assimilation nicht oder nur schwach gehemmt wird.

\begin{tabular}{|c|c|c|c|c|c|c|}
\hline \multirow[b]{2}{*}{ MJ-Konzentration } & \multicolumn{3}{|c|}{ Chlorella pyrenoidosa } & \multicolumn{3}{|c|}{ Chlorella elipsoidea } \\
\hline & Kontrolle & $10^{-6}-m$. & $10^{-5}-m$ & Kontrolle & $10^{-5}-m$ & $3 \cdot 10^{-5}-m$. \\
\hline $\begin{array}{l}\text { Gesamtfixierung } \\
\text { in \% der Kontrolle } \\
\text { Unlöslich in \% der }\end{array}$ & 100 & 29 & 2 & 100 & 25 & 8 \\
\hline Gesamtfixierung & 44 & 20 & 24 & 64 & 51 & 26 \\
\hline Löslich & 66 & 80 & 76 & 36 & 49 & 74 \\
\hline Phosphatester & 3 & 23,6 & 3 & 4 & 10 & 6 \\
\hline Saccharose & 48 & 25 & 8 & 25,5 & 6,6 & 5,5 \\
\hline Alanin & 1 & 9,5 & 25 & 1 & 16 & 28,5 \\
\hline Asparaginsäure & 0,4 & 5,1 & 4,0 & 0,25 & 1,5 & 3,2 \\
\hline Glutaminsäure & 1,0 & 9,0 & 9,0 & 0,6 & 4,5 & 6,0 \\
\hline Citrullin & 0,1 & 1,7 & 13,0 & 1,4 & 3,0 & 4,0 \\
\hline Glycocoll u. Serin & 0,8 & 4,0 & 12,0 & 1,6 & 6,0 & 9,3 \\
\hline Äpfelsäure & 0,1 & 0,8 & 1,0 & 0,2 & 0,3 & 0,4 \\
\hline Verschiedenes & 1,7 & 1,3 & 1,0 & 1,5 & 1,0 & 7,4 \\
\hline
\end{tabular}

Tab. 5. Verteilung der Radioaktivität nach 1-stdg. Photosynthese in ${ }^{14} \mathrm{CO}_{2}$ im Anschluß an 2 Stdn. Vorvergiftung.

15 D. J. Arnon, M. B. Allen u. F. R. Whatlex, Biochim. biophysica Acta [Washington] 20,449 [1956]. 


\begin{tabular}{|c|c|c|c|c|c|}
\hline & & $\begin{array}{l}\text { Kontrolle } \\
60 \text { sec PS }\end{array}$ & $\begin{array}{c}10^{-6}-m . \mathrm{MJ} \\
60 \mathrm{sec} \text { PS }\end{array}$ & $\begin{array}{c}3 \cdot 10^{-6}-m . \text { MJ } \\
60 \text { sec PS }\end{array}$ & $\begin{array}{c}10^{-5}-m . \mathrm{MJ} \\
60 \text { sec PS }\end{array}$ \\
\hline \multicolumn{2}{|c|}{$\begin{array}{l}\text { Gesamtfixierung } 10^{6} \mathrm{IPM} \\
\text { Unlöslich in \% der Gesamtfixierung } \\
\text { Saccharose } \\
\text { Diphosphate } \\
\text { Monophosphate }\end{array}$} & $\begin{array}{r}5,7 \\
29,8 \\
1,5 \\
5,6 \\
49,0\end{array}$ & $\begin{array}{r}5,1 \\
27,4 \\
1,7 \\
6,3 \\
43,3\end{array}$ & $\begin{array}{r}2,15 \\
20,4 \\
1,4 \\
1,6 \\
52,8\end{array}$ & $\begin{array}{c}0,62 \\
11,2 \\
1,4 \\
0,1 \\
53,8\end{array}$ \\
\hline 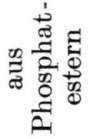 & $\begin{array}{l}\text { Glucose }+ \text { Heptose } \\
\text { Fructose } \\
\text { Pentose } \\
\text { Glycerinsäure }\end{array}$ & $\begin{array}{r}21,0 \\
5,5 \\
5,0 \\
23,0\end{array}$ & $\begin{array}{r}11,0 \\
3,6 \\
5,0 \\
30,0\end{array}$ & $\begin{array}{r}8,4 \\
2,5 \\
0,5 \\
43,0\end{array}$ & $\begin{array}{r}3,6 \\
1,2 \\
0,1 \\
49,0\end{array}$ \\
\hline \multicolumn{2}{|c|}{$\begin{array}{l}\text { PEB } \\
\text { Aminosäuren } \\
\text { org. Säuren }\end{array}$} & $\begin{array}{l}2,8 \\
9,1 \\
1,5\end{array}$ & $\begin{array}{r}3,8 \\
14,0 \\
3,5\end{array}$ & $\begin{array}{r}3,5 \\
17,0 \\
3,0\end{array}$ & $\begin{array}{r}1,0 \\
20,0 \\
11,0\end{array}$ \\
\hline
\end{tabular}

Tab. 6. Verteilung der Radioaktivität bei Chlorella elipsoidea nach 1 min Photosynthese in ${ }^{14} \mathrm{CO}_{2}$ im $\mathrm{Anschluß}$ an eine 2 -stdg. Vorvergiftung mit MJ.

Weiterhin wurde eine Reihe von kurzfristigen Fixierungen von ${ }^{14} \mathrm{CO}_{2}$ ausgeführt. Auch dabei waren die Algen $2 \mathrm{Stdn}$. mit der entsprechenden Konzentration vorvergiftet. Sie wurden dann in einen Lollipop gegeben, $10 \mathrm{~min}$ unter Durchleiten von Luft mit $0,1 \% \mathrm{CO}_{2}$ mit rund 30000 Lux belichtet, dann mit $3 \mu$ Molen $\mathrm{KH}^{14} \mathrm{CO}_{3}$ versetzt und nach der entsprechenden Zeit in $70 \%$ Alkohol (Endkonzentration) abgetötet.

Die Tab. 6 zeigt einen Versuch, bei dem mit Chl. elipsoidea jeweils $60 \mathrm{sec}$ unter Zusatz verschiedener MJ-Konzentrationen im Licht ${ }^{14} \mathrm{CO}_{2}$ fixiert wurde. Es ergibt sich ein ganz ähnliches Bild wie bei den oben beschriebenen längeren Versuchen. Mit steigender Konzentration wird die Zuckersynthese gehemmt und die restliche Aktivität findet sich in PGS und deren Folgeprodukten (Aminosäuren und organische Säuren). Bemerkenswert ist, daß bei der nur schwach hemmenden Konzentration von $10^{-6}-m$. MJ das Ribolusediphosphat (RuDP) (über 90\% der angeführten Pentose ist Ribulose) im Gegensatz zu den Hexosephosphaten noch keinerlei Abnahme zeigt, während die Zunahme der Aktivität in PGS und den Säuren schon deutlich ist. Der Aktivitätsanteil der Saccharose, der in diesen Kurzversuchen noch recht klein ist, zeigt nur eine schwache Hemmung. Offensichtlich wird der wenige, noch gebildete Zucker rasch in diesem Reservoir abgelagert.

In der Tab. 7 sind einige Versuche zusammengestellt, bei denen die Gesamtfixierung sowohl bei

\begin{tabular}{|c|c|c|c|c|c|c|c|c|}
\hline \multirow{2}{*}{\multicolumn{2}{|c|}{ Zeit [sec] }} & \multicolumn{4}{|c|}{ Kontrolle } & \multicolumn{3}{|c|}{$5 \cdot 10^{-6}-m . \mathrm{MJ}$} \\
\hline & & $\begin{array}{c}\text { volles } \\
\text { Licht } \\
30\end{array}$ & $\begin{array}{l}50 \% \\
\text { Licht } \\
30\end{array}$ & $\begin{array}{l}25 \% \\
\text { Licht } \\
30\end{array}$ & $\begin{array}{l}25 \% \\
\text { Licht } \\
15\end{array}$ & $\begin{array}{c}\text { volles } \\
\text { Licht } \\
60\end{array}$ & $\begin{array}{l}25 \% \\
\text { Licht } \\
60\end{array}$ & $\begin{array}{c}50 \% \\
\text { Licht } \\
30\end{array}$ \\
\hline \multirow{3}{*}{\multicolumn{2}{|c|}{$\begin{array}{l}\text { Gesamtfixierung } 10^{6} \text { IPM } \\
\text { Unlöslich in \% der } \\
\text { Gesamtfixierung } \\
\text { Diphosphate }\end{array}$}} & 1,2 & 1,1 & 0,53 & 0,25 & 0,68 & 0,44 & 0,42 \\
\hline & & 7,5 & 8,1 & 9,0 & 8,8 & 4,8 & 7,5 & 8,3 \\
\hline & & 9,0 & 6,0 & 7,0 & 8,2 & 1,3 & 1,0 & 1,0 \\
\hline \multirow{3}{*}{ 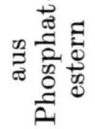 } & Glucose + Heptose & 26,6 & 31,0 & 31,0 & 20,0 & 4,3 & 2,0 & 3,0 \\
\hline & Fructose & 9,5 & 10,5 & 11,0 & 12,0 & 2,3 & 1,0 & 1,5 \\
\hline & Pentose & 6,3 & 4,5 & 4,0 & 3,0 & 1,2 & 1,0 & 0,9 \\
\hline \multicolumn{2}{|c|}{ Saccharose } & 1,4 & 2,0 & 1,1 & 1,0 & 1,1 & 0,5 & 0,5 \\
\hline \multicolumn{2}{|c|}{ P-Glycerinsäure } & 26,1 & 26,0 & 29,0 & 35,0 & 46,0 & 52,0 & 55,0 \\
\hline \multicolumn{2}{|c|}{$\mathrm{PEB}$} & 2,0 & 2,5 & 3,2 & 5,5 & 3,0 & 3,8 & 4,1 \\
\hline \multicolumn{2}{|c|}{ Aminosäuren } & 13,0 & 9,0 & 7,0 & 9,0 & 18,0 & 15,5 & 14,0 \\
\hline \multicolumn{2}{|c|}{ org. Säuren } & 6,5 & 6,0 & 6,0 & 4,5 & 19,0 & 14,0 & 12,0 \\
\hline
\end{tabular}

Tab. 7. Verteilung der Radioaktivität nach kurzfristiger Photosynthese bei Chlorella elipsoidea in ${ }^{14} \mathrm{CO}_{2}$ im $\mathrm{Anschluß}$ an eine 2 -stdg. Vorvergiftung. $m / 100$-Phosphatpuffer $p_{\text {H }} 5,5$; volles Licht $=30000$ Lux auf beiden Seiten eines Lollipop. Die Lichtstufungen wurden durch Vorschalten entsprechender Absorptionsgitter hergestellt. 
den Kontrollen als auch bei den vergifteten Algen durch Variation der Zeit und der Lichtintensität verändert wurde. Volles Licht ergab bereits gute Lichtsättigung, wie das Gleichbleiben der Gesamtfixierung bei Vorschalten eines Gitters mit 50\% Absorption zeigte. Die Hemmung der Gesamtfixierung durch MJ betrug rund 60 Prozent. Vergleicht man die beiden Gruppen, so zeigt sich, daß unabhängig von den verschiedenen Zeit- und Lichtstärkekombinationen die vergifteten Algen immer eine starke Hemmung der Zuckersynthese aufweisen und die Hauptmenge der Aktivität in den Säuren zu finden ist.

\section{b) Lokalisierung der MJ-Hemmung durch Fütterung markierter Glucose im Licht}

Mit Proben der gleichen Algensuspension, mit der die oben beschriebenen Fütterungen von markierter Glucose im Dunkeln vorgenommen wurden, sind auch Lichtversuche ausgeführt worden (Tab. 3). Dabei wurden die Algen jeweils $10 \mathrm{~min}$ vor Zugabe der Glucose mit Luft $+0,1 \% \quad \mathrm{CO}_{2}$ durchspült und mit rund 30000 Lux belichtet.

Vergleicht man die Gesamtassimilation, so wurden bei den Kontrollen entsprechend den früheren Befunden ${ }^{17}$ im Licht $30 \%$ mehr Glucose assimiliert als im Dunkeln. Außerdem ging ein erheblich größerer Teil in das Polysaccharid. Weiterhin ist bemerkenswert, daß die Pentose einen vielfach höheren Aktivitätsanteil aufweist. Es handelt sich dabei vorwiegend um Ribulosediphosphat, während im Dunkeln praktisch nur Ribose-Phosphat vorliegt.

Die für die Photosynthese sehr stark giftige MJKonzentration von $10^{-4}-m$. führt im Licht im Gegensatz zum Dunkeln zu keiner Hemmung der Glucoseassimilation, wie bereits früher ausführlicher dar- gestellt wurde ${ }^{14}$. Der größte Teil der Aktivität findet sich bei der Kontrolle im Polysaccharid. Der wesentlichste Unterschied zur Kontrolle ergibt sich bei der PGS. Während im Dunkeln der Aktivitätsanteil der PGS unter MJ stark abnimmt, steigt er im Licht an.

Wegen der Hemmung der Triosephosphatdehydrase ist es unwahrscheinlich, daß die vermehrte Aktivität in der PGS über die Oxydation des Triosephosphats dorthin gelangt. Der gleichbleibende Anteil des RuDP macht es dagegen wahrscheinlich, daß die markierte Glucose über den Pentosephosphatzyklus zum RuDP umgebaut und durch Carboxylierung und Spaltung der Ketosäure in PGS verwandelt wird. Für diese Vorstellung spricht auch der frühere Befund ${ }^{18}$, wonach exogene Glucose sehr gut in den Photosynthesezyklus einbezogen wird und durch Verdünnung des $\mathrm{CO}_{2}$-Acceptors (RuDP) zur dauernden Aufrechterhaltung der Ungleichverteilung innerhalb der Hexose bei Photosynthese in ${ }^{14} \mathrm{CO}_{2}$ führt.

Ein weiterer Unterschied gegenüber der Wirkung der MJ im Dunkeln ist das Fehlen der starken Erhöhung des Aktivitätsanteils des FDP. Auch dieser Effekt deutet darauf hin, daß im Licht der Weg zum RuDP trotz MJ möglich ist und somit die Hemmung des Hexoseabbaues auf der Triosephosphatstufe umgangen wird.

\section{c) Lokalisierung der MJ bei der Photosynthese durch Bestimmung der Zwischenstoff-Konzentra- tionen im Licht}

Die Tab. 8 zeigt die Zwischenstoff-Konzentrationen von Algen, die nach 15 min Belichtung 60 min mit verschiedenen Konzentrationen MJ unter Durchspülung mit Luft $+4 \% \mathrm{CO}_{2}$ im Licht inkubiert wurden. Die PGS ist leicht erhöht, aber die im Dunkeln

\begin{tabular}{|c|c|c|c|c|}
\hline \multirow{2}{*}{ Zwischenstoff } & \multirow{2}{*}{65 min Licht } & \multicolumn{2}{|c|}{ Nach 15 min Licht, 60 min MJ im Licht } \\
\cline { 2 - 5 } & & $10^{-4}-m$. MJ & $3 \cdot 10^{-5}-m$. MJ & $10^{-5}-m$. MJ \\
\hline ATP & 5,10 & 5,14 & 4,94 & 5,40 \\
ADP & 3,78 & 3,77 & 3,76 & 25,76 \\
PGS & 22,90 & 26,30 & 0,75 & 0,755 \\
FDP & 0,72 & 0,78 & 1,44 & 1,51 \\
GAP & 1,44 & 1,64 & 0,82 & 0,82 \\
BTS & 0,94 & 1,28 & 1,85 & 1,71 \\
\hline
\end{tabular}

Tab. 8. Veränderung der Zwischenstoff-Konzentrationen bei Chlorella pyrenoidosa nach Zugabe von MJ im Licht. Gasphase Luft $+4 \% \mathrm{CO}_{2}, m / 30$-Phosphatpuffer $p_{\mathrm{H}} 5,5$. Werte $\cdot 10^{-4}-m$.

16 H. A. Krebs u. K. Henseleit, Hoppe-Seyler's Z physiol. ${ }^{17}$ O. Kandler, Z. Naturforschg. 9 b, 625 [1954]. Chem. 210, 33 [1932]. 
beobachtete Anreicherung des FDP fehlt völlig. Ähnlich wie bei der Verteilung der Radioaktivität nach Fütterung markierter Glucose bewirkt MJ im Licht kaum eine Veränderung.

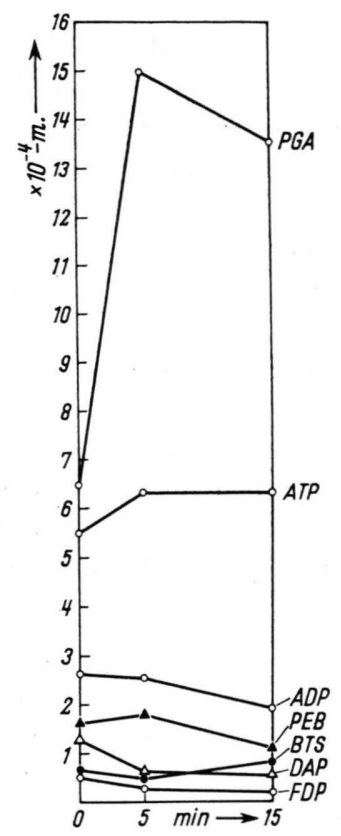

Abb. 2. Veränderung der Zwischenstoff-Konzentrationen bei Chlorella pyrenoidosa nach Zugabe von $10^{-3}-m$. MJ zu belichteten Suspensionen. Vor Giftzugabe 1 Stde. mit Glucose inkubiert. $m / 30$-Phosphatpuffer $p_{\mathrm{H}} 5,5$.

Auch bei dem in Abb. 2 dargestellten Versuch, bei dem analog zu Abb. 1 eine sehr hohe Konzentration von MJ im Licht gegeben wurde, sind die Veränderungen relativ schwach und betreffen hauptsächlich einen leichten Anstieg der PGS, BTS und PEB, während FDP leicht abfällt. Die Wirkung des Lichtes auf den durch MJ gehemmten Stoffwechsel läßt sich am besten in Abb. 3 ablesen. Dabei wurde ganz ähnlich wie in Abb. 1 nach 1 Stde. Glucosefütterung im Dunkeln $10^{-3}-m$. MJ zugegeben und nach 30 min die Restsuspension belichtet und weiterhin Proben entnommen. In wenigen min verschwinden FDP und DAP, während PGS und die Folgeprodukte entsprechend zunehmen. Auch ATP zeigt einen raschen Anstieg.

Dieser Versuch bestätigt die aus den Experimenten mit markierter Glucose gezogenen Schlußfolgerungen im vollen Umfang und kann nur so verstanden werden, daß im Licht FDP über RuDP zu PGS verwandelt wird. Da die Reduktion der PGS bzw. deren Muttersubstanz (Ketosäure) zu Zucker-

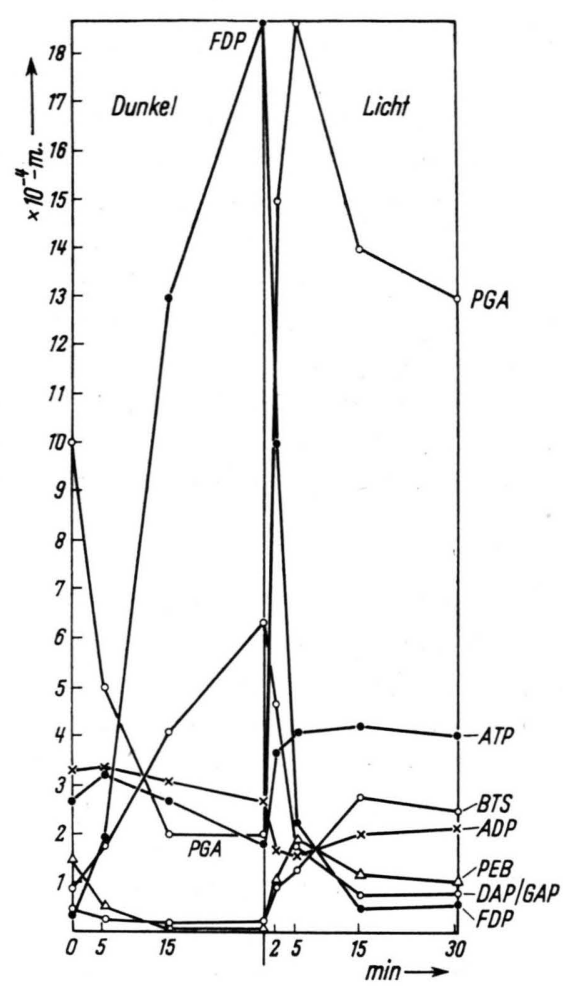

Abb. 3. Veränderung der Zwischenstoff-Konzentrationen bei Chlorella pyrenoidosa nach Zugabe von $10^{-3}-m$. MJ im Dunkeln und Übergang zu Licht 30 min nach der Vergiftung. 1 Stde. vor Giftzugabe mit Glucose inkubiert. $m / 30$-Phosphatpuffer $p_{\mathrm{H}} 5,5$. Gasphase Luft $+4 \% \mathrm{CO}_{2}$.

phosphat durch MJ blockiert ist, reichert sie sich an und führt auch zu verstärkter Bildung von BTS und anderer organischer Säuren.

\section{Diskussion der Ergebnisse}

Sowohl die Veränderungen der ZwischenstoffKonzentrationen als auch die Verteilung der Radioaktivität nach Fütterung markierter Glucose sprechen dafür, daß auch bei Chlorella die Hemmung der Triosephosphatdehydrogenase die erste nachweisbare Wirkung von MJ auf die Atmung darstellt. Weiterhin zeigen die Versuche, daß trotz der Hemmung der Glykolyse noch genügend ATP zur Verfügung steht, um FDP in ungewöhnlich hohen Konzentrationen anzureichern.

Da die zur Umwandlung der Hexose in Pentose nötigen Transaldolase-, Transketolase und Epimerase-Reaktionen energetisch neutral sind und die Ru5-P-Kinase eine sehr hohe Affinität zu ATP besitzt (Mi c h a e lis - M e n t e n - Konstante der Fructose- 
6-Phosphatkinase für ATP $2,4 \cdot 10^{-3} 19$, die der Ru5-P-Kinase für ATP jedoch nur $2,8 \cdot 10^{-420}$ ), wäre zu erwarten, daß RuDP gebildet wird. Dies ist jedoch im Dunkeln nicht der Fall, wie die Fütterung radioaktiver Glucose zeigt. Schon die Untersuchungen Calvins (vgl. l. c. ${ }^{1}$ ) hatten ergeben, daß im Dunkeln praktisch kein RuDP in der Zelle vorhanden ist, und auch die gute ATP Versorgung bei der Glucoseatmung, die die verschiedensten Synthesewege ermöglicht, führt nicht zur Bildung von RuDP (Tab. 3).

Dagegen wird im Licht sofort RuDP synthetisiert und es bildet sogar den größten „pool“ aller Zuckerphosphate ${ }^{1}$. Selbst bei sehr drastischer Vergiftung mit MJ wird das in einer vorhergehenden Dunkelperiode angestaute FDP im Licht rasch zu RuDP und weiter zu PGS verwandelt, obwohl nach den Befunden von Calo und GibBs ${ }^{5}$ die Ru-5-P-Kinase durch die verwendete Konzentration praktisch völlig gehemmt sein müßte. Die Bildung von RuDP scheint demnach ein extrem lichtabhängiger Vorgang zu sein, der nach den bisher bekannten Tatsachen nicht einfach durch Konzentrations-Änderungen der ATP gesteuert sein kann.

Eine widerspruchsfreie Erklärung kann gegeben werden, wenn man annimmt, daß das primär bei der Lichtphosphorylierung entstehende energiereiche Phosphat $\left(\left[\sim p_{\mathrm{H}}\right]\right)$ nicht obligat auf das Adenylsäuresystem übertragen wird, sondern direkt zur Phosphorylierung von Ru-5-P zu RuDP verwendet werden kann, wie das Schema der Abb. 4 zeigt. Die Reaktion könnte außerdem von Ru-5-P-Kinase kata-

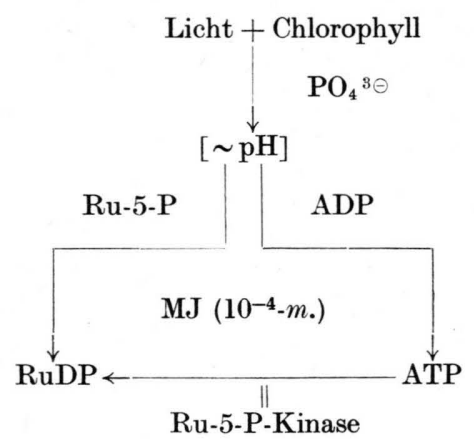

Abb. 4. Schema für die Verwendung des im Licht gebildeten energiereichen Phosphats.

18 M. Gibbs u. O. Kandeer, Proc. nat. Acad. Sci. USA 43, 446 [1957].

19 B. Axelrod, P. Saltman, R. S. Bandurski u. R. S. Baker, J. biol. Chemistry 197, 89 [1952].

20 J. Hurwitz, A. Weissbach u. B. L. Horecker, J. biol. Chemistry 218, 764 [1956]. lysiert werden, nur müßte man eine wesentlich höhere Affinität zu $\left[\sim p_{\mathrm{H}}\right]$ annehmen, als sie für ATP besteht. Damit würde auch die Empfindlichkeit gegen MJ geringer sein als bei den Versuchen von Calo und GibBs ${ }^{5}$, die das Ferment mit ATP koppelten.

Einen weiteren Hinweis auf die Umgehung des Adenylsäuresystems bei der Bildung von RuDP mit Hilfe der Lichtphosphorylierung stellen die Befunde von WEICHART ${ }^{21}$ dar, die er bei Fütterung von ${ }^{32} \mathrm{P}$ zu Elodeablättchen erhob. Die einzige Verbindung, die mit Verkürzung der Photosynthesezeit eine starke Zunahme des prozentualen Anteils der aufgenommenen Radioaktivität zeigte, war RuDP, während die Aktivitätsanteile von ATP ebenso wie die der Zuckerphosphate und PGS bei kurzen Zeiten gegen Null gingen. Wäre ATP der Vorläufer des RuDP, so müßte sich für ATP eine Kurve ergeben, wie sie tatsächlich für RuDP gefunden wurde.

Einen ersten experimentellen Beweis für die Existenz eines nicht mit ATP identischen energiereichen Phosphats bei der Lichtphosphorylierung stellen vielleicht die neueren Befunde von KAHN und JAGENDORF ${ }^{24}$ mit isolierten Chloroplasten dar, die allerdings noch weiterer Ergänzung bedürfen.

Die Anreicherung von PGS und die geringe Radioaktivität in den Zuckerphosphaten nach der MJ-Hemmung der Photosynthese sprechen zunächst dafür, daß genau wie bei der Atmung die Hemmung der Triosephosphatdehydrogenase der primäre Angriffspunkt der MJ ist. Dagegen spricht jedoch die um 2 Zehnerpotenzen größere Empfindlichkeit der Photosynthese, die schon von Konzentrationen gehemmt wird, bei denen die Glykolyse und die Bildung von Zucker aus Pyruvat in Umkehr der Glykolyse ( $\mathrm{O}_{A K S}$ und KANDLER ${ }^{22}$ ) noch unbeeinflußt sind. Es ergibt sich folgende Reihenfolge der Empfindlichkeit gegenüber $\mathrm{MJ}$ : Photosynthetische $\mathrm{CO}_{2}$-Reduktion $>$ Ru-5-P-Kinase $>$ Triosephosphatdehydrogenase.

Schon früher hatten wir ${ }^{23}$ auf Grund anderer Befunde angenommen, daß bei der Photosynthese unter Umgehung der PGS die aus der Carboxylierung des RuDP entstehende Ketosäure direkt reduziert und zur Hexose isomeriert werden kann. PGS

21 G. WeIchart, Dissertation, Tiermedizinische Fakultät, Hannover 1958.

22 B. A. OAKs u. O. Kandler, Phys. Plant., im Druck.

23 O. Kandler, Z. Naturforschg. 12 b, 271 [1957].

24 J. S. KaHN u. A. T. Jagendorf, Biochem. Biophys. Res. Communications 2, 259 [1960]. 


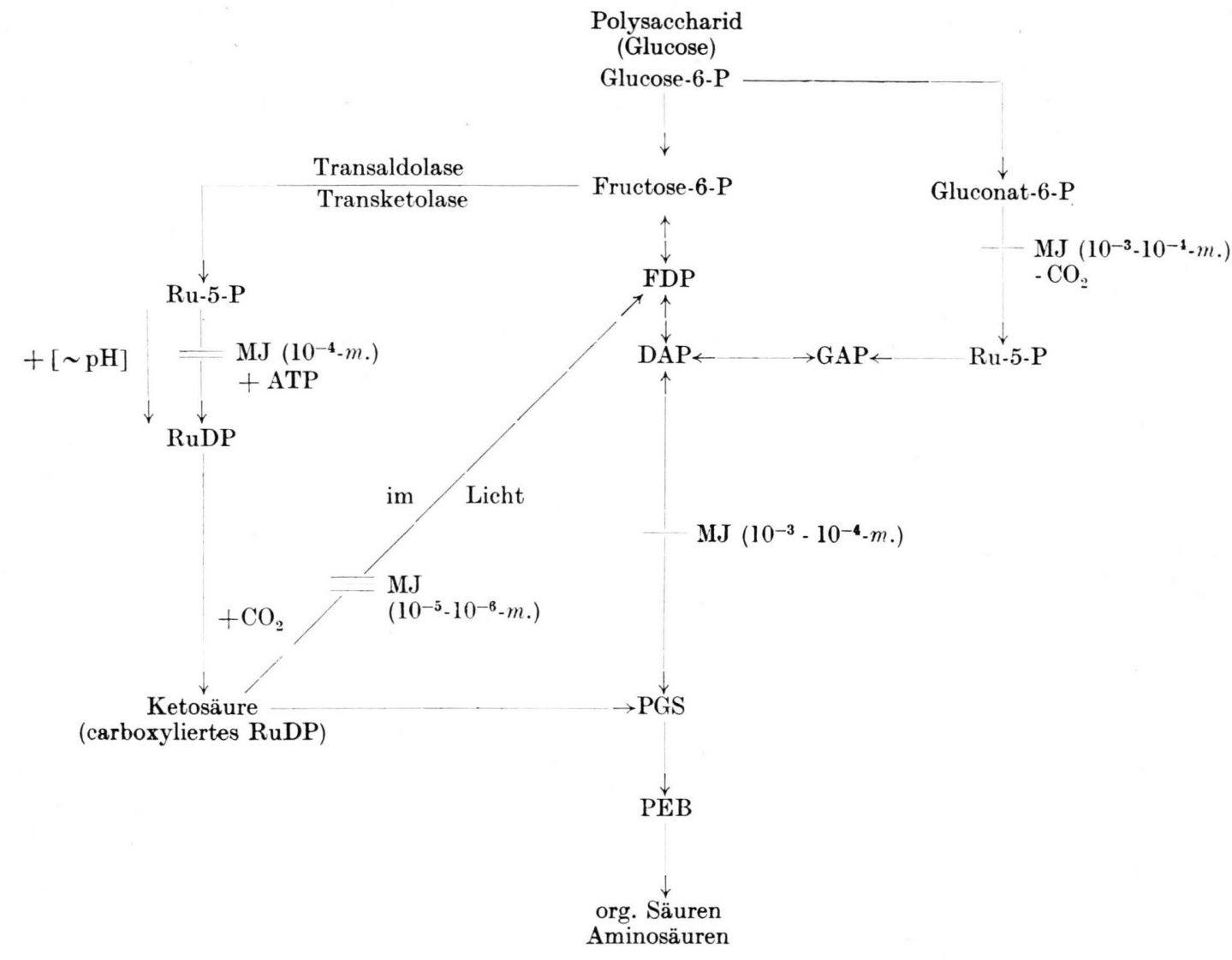

Abb. 5. Darstellung der Angriffspunkte von MJ bei Photosynthese und Atmung. Die angegebenen Konzentrationsbereiche führen zu rund $50 \%$ Hemmung der betreffenden Reaktion.

entsteht dann nur bei mangelndem Reduktionsvermögen in größerem Umfang aus der Ketosäure. Es ist vorstellbar, daß die Reduktion der Ketosäure eine besonders hohe Empfindlichkeit gegen MJ aufweist. Entsprechend den im Schema der Abb. 5 dargestellten Verhältnissen müßte sich bei MJ-Vergiftung PGS anreichern, da auf Grund der nicht gehemmten Lichtphosphorylierung reichlich RuDP aus den Zuckerreserven synthetisiert wird, das nach Carboxylierung nicht reduziert, sondern zu PGS gespalten wird. Letztere fließt dann über BTS zu organischen Säuren ab und führt auch zu den schon von HoLzer ${ }^{13}$ beschriebenen Atmungssteigerungen nach Belichtung vergifteter Chlorellen. Nur in sehr geringem Umfang, entsprechend der Zuckersynthese bei BTS-Fütterung, kann die PGS in Umkehr. der

* Die Angabe der Autoren ${ }^{25}$, daß sie rund 25\% der Photosynthese-Aktivität der Blätter in den Chloroplasten wiederfanden, beruht nicht auf einer hohen Photosyntheserate der Chloroplasten, sondern auf extrem niederen Werten für die Blätter (16 bis $30 \mu \mathrm{m} \mathrm{CO} / \mathrm{h} \cdot \mathrm{mg}$ Chlorophyll). Da keine genauen methodischen Angaben über die Photo- wesentlich weniger MJ-empfindlichen Glykolyse zu Zucker reduziert werden.

Diese im Vergleich zur Photosynthese geringe Rate der Zuckerbildung in Umkehr der Glykolyse scheint auch in den von Trebst und Mitarbb. ${ }^{6}$ benützten "gebrochenen Chloroplasten“ verwirklicht zu sein, denn bei diesen Versuchen wurde die $\mathrm{CO}_{2}$ Fixierung bei Zugabe von RuDP erst mit sehr hohen MJ-Konzentrationen gehemmt. Außerdem war im Gegensatz zu den Verhältnissen bei ganzen Zellen die Bildung von RuDP stärker gehemmt als die Reduktion. Auch die geringen Raten der $\mathrm{CO}_{2}$-Fixierung dieses Systems von nur 1 bis $3 \mu$ Molen $\mathrm{CO}_{2} / \mathrm{mg}$ Chlorophyll $\cdot$ Stde. sprechen dafür, daß die in vivo ablaufenden Photosyntheseprozesse in den gebrochenen Chloroplasten nicht erhalten geblieben sind *

synthese-Messung in den Blättern gemacht wurden, kann man über die Ursachen der geringen Aktivität der Blätter nur Vermutungen anstellen (ungenügende Belichtung der in den Blättern dicht gepackten Chloroplasten; Spaltöffnungsschluß wegen zu hoher $\mathrm{CO}_{2}$-Konzentration in den Versuchsgefäßen usw.). 
Wie schon kurz mitgeteilt ${ }^{8}$, führen die Phosphorylierungsgifte Arsenat, Azid und Dinitrophenol bei Chlorella nicht zu einer Anreicherung von PGS, sondern hemmen die Bildung von RuDP, während Trebst und Mitarbb. ${ }^{6}$ bei gebrochenen Chloroplasten das Gegenteil, nämlich die Anreicherung von PGS, fanden. Diese offensichtliche Diskrepanz kann am einfachsten mit der Annahme erklärt werden, $\mathrm{da} \beta$ den gebrochenen Chloroplasten die beiden typischen Photosynthese-Reaktionen, Phosphorylierung des Ru-5-P mit $\left[\sim p_{\mathrm{H}}\right]$ und Reduktion der Keto-

25 M. Losada, A. V. Trebst u. D. I. Arnon, J. biol. Chemistry 235, 832 [1960]. säure, fehlen und durch die ATP gekoppelte Bildung von RuDP bzw. die Reduktion von PGS ersetzt werden. Weitere Untersuchungen, insbesondere Versuche mit ganzen Chloroplasten hoher Photosynthese-Aktivität scheinen uns notwendig zu sein, um zu klären, ob die bisher aufgetretenen Widersprüche tatsächlich Anzeichen verschiedener Reaktionsabläufe sind oder nur auf methodischen Differenzen beruhen.

Die Arbeit wurde von der U.S. A ir F o r c e unter Contract No. AF 61 (052)-244 gefördert.

Der Deutschen Forschungsgemeins chaft danken wir für Gewährung einer Sachbeihilfe.

\title{
Vergleichende Untersuchungen über die antigene Wirksamkeit verschiedener gereinigter Serumprotein-Fraktionen ${ }^{*}$
}

II. Mitt.: Humanalbumin sowie $\gamma$-Globulin vom Rind als Antigene. Präzipitat-N-Bestimmung nach Heidelberger-Kendall

\author{
Von H. Götz, F. Scheiffarth und Ch. Zimmer \\ Aus der Medizinischen Universitätsklinik Erlangen \\ (Z. Naturforschg. 16 b, 61—67 [1961] ; eingegangen am 15. März 1960)
}

\begin{abstract}
In Fortsetzung früherer Studien über die Antigenität verschiedener proteiner Antigene wurden unter Verwendung der $\mathrm{He}$ idelberger-Kendall-Technik und der Papierelektrophorese Humanalbumin und Rinder- $\gamma$-Globulin im Rahmen einer tierexperimentellen Versuchsserie untersucht. Hierbei wurden folgende Ergebnisse erzielt :

1. In Gruppe A (mit Humanalbumin sensibilisiert) trat, im Gegensatz zu Gruppe B (mit $\gamma$-Globulin vom Rind sensibilisiert), die Antikörperbildung zu einem späteren Zeitpunkt ein; auch war die gebildete Präzipitinmenge geringer als in Gruppe B.

2. Zwischen der Menge des applizierten Antigens und der Menge des gebildeten Antikörpers besteht eine direkte Abhängigkeit. In Gruppe B wurde pro mg des applizierten Antigens mehr Präzipitin gebildet als in Gruppe A.

3. Es bestehen Beziehungen zwischen der applizierten Antigenmenge und dem beobachteten $\gamma$-Globulinzuwachs sowie zwischen dem $\gamma$-Globulinzuwachs und der Menge der gebildeten Antikörper.

4. Die Ergebnisse der vorgenommenen Präzipitatüberstands-Bestimmungen bestätigen die ermittelten Präzipitatoptima.

In der Diskussion wird im wesentlichen auf die unterschiedlichen physikalischen, chemischen und biologischen Eigenschaften der verwendeten Antigene hingewiesen, die ursächlich für die unterschied liche Antikörperbildung verantwortlich gemacht werden.
\end{abstract}

Unter Bezugnahme auf die 1. Mitteilung dieser Problemstellung ${ }^{12}$ werden im folgenden die Ergebnisse der Präzipitat-N-Bestimmung nach $\mathrm{H}$ e i d e lberger-Kendall vorgelegt und diskutiert. Es war beabsichtigt, vor allem quantitative und zeitliche Unterschiede in der Antikörperbildung der verschiedenen, hier angewandten Antigene zu erfassen. Außerdem sollte das Verhalten der Serum- $\gamma$-Globuline an Hand der papierelektrophoretisch gewonne-

\footnotetext{
* Unter Verwendung verschiedener Antikörper-Nachweismethoden, insbesondere neuerer Geltechniken sowie der Präzipitat-N-Bestimmung nach $\mathrm{He}$ idelberger-Kendall.
}

nen Werte verfolgt und zur Präzipitinbildung in Beziehung gesetzt werden.

Tiermaterial, Sensibilisierungsmodus sowie Versuchsanordnung entsprachen den Angaben in der 1. Mitteilung ${ }^{* *}$.

Von den Untersuchungsmethoden waren für die vorliegende Arbeit die Gesamteiweißbestimmung, die Papierelektrophorese sowie die Präzipitat-N. Bestimmung nach $\mathrm{Heidelberger-Kendall}$

\footnotetext{
** Hier entspricht die Gruppe A (mit Humanalbumin sensibilisiert) der Gruppe I aus der 1. Mitt. und die Gruppe B (mit Rinder- $\gamma$-Globulin sensibilisiert) der Gruppe II aus der 1. Mitteilung.
} 